\title{
Student Engagement with Video-Watching and Flipped Class Behaviors
}

\author{
Shutong Xie ${ }^{1,2, \text { a }}$, Wun-Kai Yuen ${ }^{2}$, Lifeng Huang ${ }^{2}$ and Vincent To Yee $\mathrm{Ng}^{2}$, b \\ ${ }^{1}$ School of Computer Engineering, Jimei University, Xiamen 361021, China \\ ${ }^{2}$ Department of Computing, The Hong Kong Polytechnic University, Hong Kong, China \\ ashutong@jmu.edu.cn, ${ }^{\mathrm{b}}$ cstyng@comp.polyu.edu.hk
}

Keywords: Learning analytics; MOOC; Engagement; Flipped classroom.

\begin{abstract}
The concept of Massive Open Online Course (MOOC) with flipped classroom has been emerged in recent years as the arrangement has demonstrated the potential enhancement of student engagement and ultimately their academic performance. In this paper, we are interested to model student engagement based on students' video-watching behaviors and their flipped classroom involvement. The work is based on the implementation of two online courses from two institutes respectively. In our work, we first explored the relationships among content fitness, ease of use, students' engagement and learning effectiveness through statistical and data mining techniques by using tracking logs, questionnaires and academic results of students. We then found out some interesting online learning patterns of video watching time, procrastinators, flipped classrooms performance, and high/low achievers as well.
\end{abstract}

\section{Introduction}

In recent years, Massive Open Online Course (MOOC) has become increasingly popular and a hot topic globally. The trend started in North America and soon spread to Europe, Asia and Australia. Obviously, MOOC is leading a revolution of education, and this set up gathers global education resources and restructures the learning environments. The concept of MOOC has evolved into different branches and cross-over with different teaching methods during its development [1]. MOOC generally has three major characteristics, namely, massive, open and online. MOOC allows people geographically-dispersed to participate without limitation. Furthermore, forum like facility is provided on MOOC for users to interact in order to build a community for student, teacher, and teaching assistant.

Some MOOC organizers had mentioned that using MOOC only without any offline interaction, the learning performance may not be as good as expected while blended learning strategy is a possible alternative [2]. The strategy is to provide knowledge to students online, and they have the autonomy to decide their learning time, venue or speed. Later, students will attend class to reinforce their knowledge by "Flipped Classroom" arrangement [3]. In a flipped classroom, students will have the assignments and discussion topics in the class under the assistant of teachers after learning assigned lessons beforehand [4]. This can encourage students to learn in a more proactive way and also have more interactions between classmates and teachers.

In this paper, we focus on studying how students engage in online classes (through similar set up as MOOC), and the relationship between their engagement and learning performance. We also work on the similarities and differences for student learning behavior of the two online courses from two institutes. Our work has two objectives. The first goal is to assess learning effectiveness under flipped classroom and MOOC settings. Then, we aim to identify activity behavior in the MOOC and flipped classroom setting.

\section{Research Methodology}

The goal of our work is to design an engagement metric which can be used to predict the learning performance of a student. A potential application is to assist teachers/mentors to receive alerts of 
at-risk students. Also, it can be used to identify the improvement spot in the teaching arrangement through learning behavior analysis. With this goal, we have two objectives which guide us to develop the engagement metric. The objectives are

- To assess learning effectiveness under flipped classroom and MOOC setting.

- To identify student activity behavior in the MOOC and flipped classroom setting.

For the first objective, we have adopted to study five factors and their related hypothesis. The five factors include the content fitness and ease of use, the engagement in online learning platform, the self-evaluated background knowledge, the learning outcome, and satisfaction towards the online platform and flipped classroom. In [5], Piccoli, Ahmad \& Ives adopted the satisfaction and performance as the learning effectiveness. This is the same consideration in our work; hence, the associated hypotheses of us are list as follow.

- Content fitness and ease of use are positively related to learning outcome, satisfaction and engagement.

- Students' previous knowledge is positively related to learning outcome and satisfaction, but negatively to engagement level

- Students' engagement is positively related to learning outcome and satisfaction

- The satisfaction is positively related to the learning outcome

There are two mediating variables, i.e. the students' engagement on the MOOC platform and satisfaction, to be studied if there is further positive correlation to the learning outcome.

For the second objective, we use the statistics analytical method in terms of two aspects, i.e. total video-watching time/duration and procrastinators who joined online class later and used it at the last day before examination. The partial least square regression is used to find the relationship between the students' video-watching time and their learning outcome. Also, the activity behavior of procrastinator can be found by correlation analysis.

\section{Course Offering and Data Acquisition}

There were two online courses participating in this study. One course was from The Hong Kong Polytechnic University and the other one was from Jimei University. Here, we tried to acquire data from the two groups of students such as video watching times, MOOC access parameter (i.e. frequency and time slot), flipped classrooms/test performance, and the final course grades as well. Our study does not only provide effective experimental review for the use of online platform in different institutes, but also help to find common effective learning patterns of student engagement.

\subsection{Database System Course for HKPU}

The selected Database Systems course is a compulsory subject for all undergraduate students studying in the Department of Computing of The Hong Kong Polytechnic University (HKPU). The main course objective is to enable students to attain a considerable complexity and understand DBMSs data models, file structure and physical database design including file organization, indexing and hashing were taught. Thirty-nine hours of lecture and thirteen hours of tutorial or laboratory with sixty-seven hours of other study effort were expected for all registered students.

In Fall 2015, two out of thirteen classes were shifted from traditional instructing mode to flipped class mode with the aid of OpenEdX as the online platform. There were three different topics being covered by the flipped classes, ERD, normalization and $\mathrm{B}+$ tree. The corresponding materials and videos for the flipped classes were published in the OpenEdX platform one week before the classes. In each flipped class, individual and group exercise were distributed for discussion and understanding the topics. After the exercises, a questionnaire was distributed to every student in each flipped class to collect their feedback. Data is acquired from the tracking log of OpenEdX, questionnaire from flipped classes, and learning results.

\subsection{Information Technology Foundation Course for JU}

The selected course was Information Technology Foundation course of Jimei University (JU), which is a compulsory subject for all undergraduate students studying in Jimei University. The 
learning contents include computer hardware and software, multimedia, database, network and information security. Twenty hours of lecture and fourteen hours of laboratory with about eight hours of other study effort were expected for all registered students. Furthermore, students were required to watch eleven short teaching videos and complete eight tests on the MOOC platform supplied by the Chaoxing Company.

The course was offered in Fall 2015. There were 4811 students registered using the MOOC platform. Students would need to complete 8 tests on the platform, but without any flipped class. The data is obtained from the tracking log of the platform, and their academic results.

\section{RESULTS and ANALYSIS}

Initially, we performed analysis of the hypothesis. Partial Least Square and Pearsons' coefficient analysis were conducted for the HKPU class; where only Pearsons' coefficient analysis was done in the JU class.

HKPU students who attended flipped classroom generally have higher activity rate in OpenEdX usage, higher expectation, better final and quiz results. These students generally had less background knowledge and positive attitude towards content fitness and ease of use in MOOC, which is shown in Table 1 . On the contrary, students who did not attend flipped class $(\mathrm{r}=-0.358, \mathrm{p}<0.005)$ and had prior knowledge, or they tended to use OpenEdX during the flipped classes ( $r=0.426, p<0.0001)$, or they felt positive about content fitness and ease of use $(r=0.279, p<0.05)$, there is no significant effect on their final and quiz results.

Therefore, encourage students to attend flipped class is important as the positive effect on online engagement, expectation, better quiz and final results. And it confirms that flipped classroom can have an association with MOOC to solve the gap of content provided by MOOC.

Table 1. Relationship results of the HKPU student who attended flipped classroom

\begin{tabular}{|l|l|l|}
\hline & \multicolumn{1}{|c|}{ Relationship Results } & \multicolumn{1}{c|}{ Correlation } \\
\hline \multirow{4}{*}{$\begin{array}{l}\text { Students who } \\
\text { attended flipped } \\
\text { classroom }\end{array}$} & Total activity rate in the OpenEdX usage & $\mathrm{r}=0.275, \mathrm{p}<0.01$ \\
\cline { 2 - 3 } & Expectation on their results & $\mathrm{r}=0.292, \mathrm{p}<0.05$ \\
\cline { 2 - 3 } & Final results & $\mathrm{r}=0.257, \mathrm{p}<0.05$ \\
\cline { 2 - 3 } & Quiz results & $\mathrm{r}=0.231, \mathrm{p}<0.05$ \\
\cline { 2 - 3 } & Less background knowledge & $\mathrm{r}=-0.358, \mathrm{p}<0.01$ \\
\cline { 2 - 3 } & $\begin{array}{l}\text { Negative attitude towards content fitness } \\
\text { and ease of use }\end{array}$ & $\mathrm{r}=-0.331, \mathrm{p}<0.01$ \\
\hline
\end{tabular}

In the review of high and low achievers of HKPU students, as shown in Table 2, high achievers normally joined the MOOC platform earlier and had higher participation before or during flipped classes. Other the other hand, low achievers were non-active users and missed the flipped classes mostly. The usage time period of them was most likely late night periods.

Table 2. Comparison between high and lower achievers for HKPU students

\begin{tabular}{|l|l|l|}
\hline & \multicolumn{1}{|c|}{ High Achievers } & \multicolumn{1}{c|}{ Low Achievers } \\
\hline OpenEdX Platform & Over 90\% joined early & Nearly 40\% are non-active users \\
\hline Usage time & $\begin{array}{l}\text { Before or during each flipped } \\
\text { class } \\
\mathrm{r}=0.306, \mathrm{p}<0.05\end{array}$ & $\begin{array}{l}\text { Overnight } \\
\mathrm{r}=0.302, \mathrm{p}<0.01\end{array}$ \\
\hline Flipped classroom & \multicolumn{1}{|c}{-} & $\begin{array}{l}\text { Not attending flipped class } \\
\mathrm{r}=-0.296, \mathrm{p}=0.01\end{array}$ \\
\hline
\end{tabular}

For JU students, high achievers accessed the MOOC more frequently, watched more videos, and completed more tasks, but access rate reduced when examination time was near. On the contrary, low achievers did less work, but most of them accessed the MOOC at very last day before exam, which is showed in Table 3. 
Table 3. Comparison between high and lower achievers for JU students

\begin{tabular}{|l|c|c|c|}
\hline & $\begin{array}{c}\text { Overall } \\
\text { (Mean) }\end{array}$ & $\begin{array}{c}\text { High Achievers } \\
\text { (Top 4\%) }\end{array}$ & $\begin{array}{c}\text { Low Achievers } \\
\text { (Low 4\%) }\end{array}$ \\
\hline Mean of frequency per person & 32.2 & 39.6 & 31.3 \\
\hline Video watching time & 198.4 & 209.7 & 190.8 \\
\hline Number of tasks (out of 19) & 17.7 & 18.2 & 17.0 \\
\hline frequency of daytime usage & 12.9 & 14.8 & 13.2 \\
\hline frequency of night usage & 17.5 & 22.7 & 16.6 \\
\hline frequency of overnight usage & 1.8 & 2.2 & 1.4 \\
\hline $\begin{array}{l}\text { frequency of 3-days before exam } \\
\text { usage }\end{array}$ & 4.2 & 1.8 & 6.6 \\
\hline
\end{tabular}

For the second objective, we studied their video watching time/duration and their learning outcomes respectively. For HKPU active students, their engagement is neutral to the final grade in general, as shown in Fig. 1 (a).

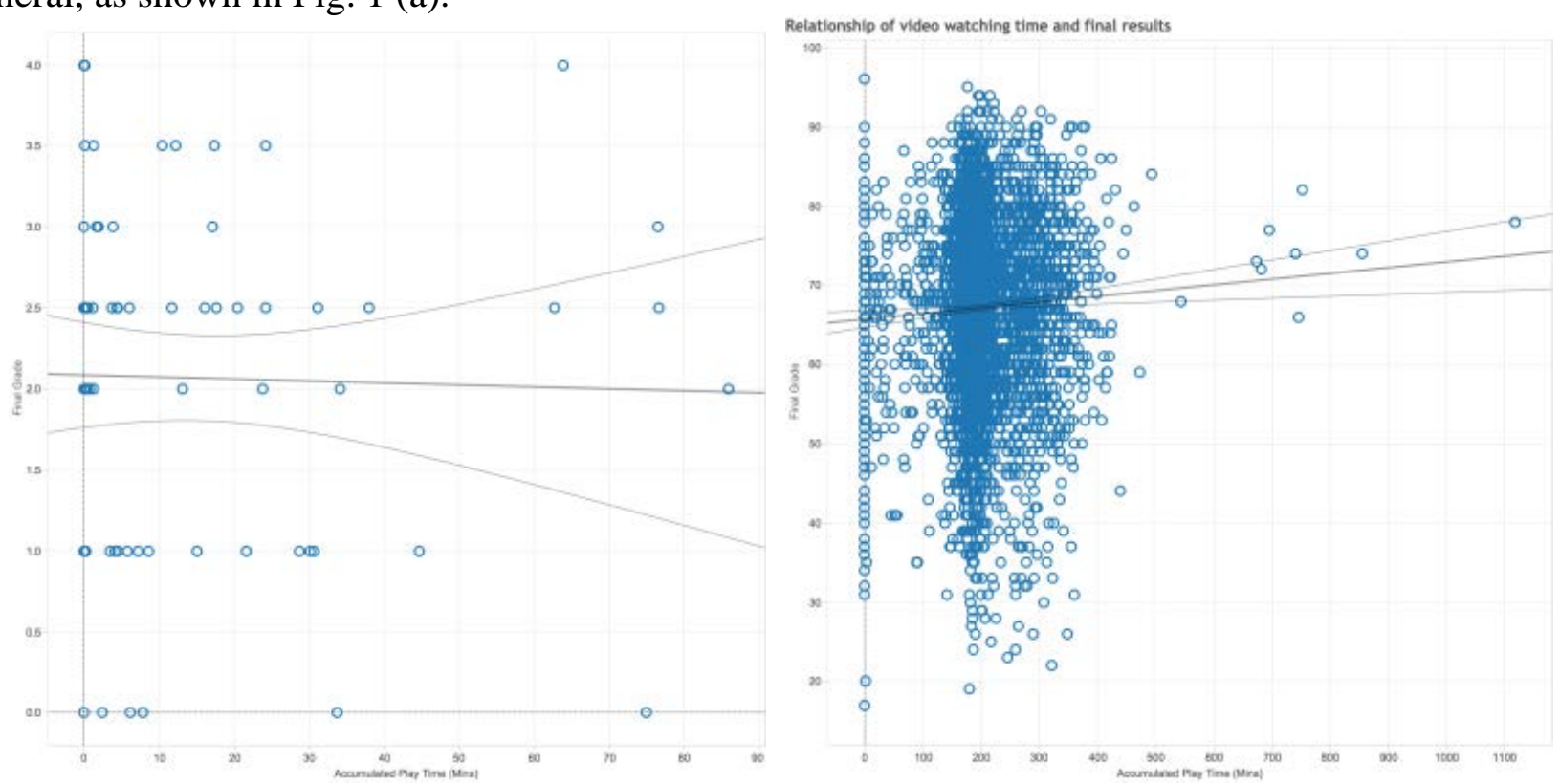

Fig. 1 Relationship between video watching time and final grade: (a) for HKPU students; (b) for JU students

Fig. 1 (b) shows all JU students' engagement in general is slightly positive to the final grade. Hence, the video watching time has different effect for the two groups of students. And it seems that the video watching time is more relevant for JU students to get a high final grade than for HKPU students.

Another interest is to review the potential procrastinators, those would use the online platform during the last day before examinations.

The procrastinators in HKPU (i.e. accessed the system 3 days before examination) tended to have lower expectation, joining MOOC late, having less activity rate and watch less videos. In the end, they achieved lower group project grade. Similarly, for Jimei university students, the procrastinators who used MOOC at very last days before examination tended to join MOOC later, and watching fewer videos. In the end, they obtain lower final grade.

In summary, we have following interesting findings:

a) Female students perform better in MOOC and flipped class settings for both college students.

b) Procrastination has negative effects towards learning performance and engagement for both college students.

c) Students with background knowledge use OpenEdX during flipped class time, but do not attend flipped class, for the Hong Kong Polytechnic University. 
d) Attending flipped classes is critical for better learning performance.

\section{Conclusions}

Students were generally having satisfactory performance for those with a high participation rate, especially the results with flipped class in HKPU. Different online behavior obviously leads to different student learning effectiveness. Some interesting online learning patterns of video watching time, procrastinators, flipped classroom, and high/low achievers for students from two institutes were found. Their online behaviors and feedback were also evaluated to strengthen students' performance and satisfaction, which can provide effective experimental review for the use of MOOC in different colleges.

In future, we plan to identify more detailed learning patterns and correlations towards learning effectiveness with a novel video watching engagement metric and extended sample size as well. On the other hand, predictive analytics would be studied, and be used to predict at-risk students in order to improve the learning outcome.

\section{References}

[1] Pappano, L., 2012. The Year of the MOOC. The New York Times 2, 12, 2012.

[2] Bruff, D.O., Fisher, D.H., Mcewen, K.E., and Smith, B.E., 2013. Wrapping a MOOC: Student perceptions of an experiment in blended learning. Journal of Online Learning and Teaching 9, 2, 187.

[3] Alonso, F., López, G., Manrique, D., and Viñes, J.M., 2005. An instructional model for web based e - learning education with a blended learning process approach. British Journal of educational technology 36, 2, 217-235.

[4] Clark, K.R., 2015. The Effects of the Flipped Model of Instruction on Student Engagement and Performance in the Secondary Mathematics Classroom. Journal of Educators Online 12, 1, 91-115.

[5] Piccoli, G., Ahmad, R., and Ives, B., 2001. Web-based virtual learning environments: A research framework and a preliminary assessment of effectiveness in basic IT skills training. MIS quarterly, 401-426. 